\title{
Application of Corvis ST to evaluate the effect of femtosecond laser-assisted cataract surgery on corneal biomechanics
}

\author{
YINJUAN WEI ${ }^{*}$, LINGXIAO XU* and HUI SONG \\ Tianjin Eye Hospital, Tianjin Key Laboratory of Ophthalmology and Visual Science, Tianjin Eye Institute, \\ Clinical College of Ophthalmology, Tianjin Medical University, Tianjin 300020, P.R. China
}

Received March 2, 2017; Accepted June 8, 2017

DOI: $10.3892 /$ etm.2017.4675

\begin{abstract}
The aim of the present study was to evaluate the effects of femtosecond laser-assisted cataract surgery (FLACS) and phacoemulsification on corneal biomechanics using corneal visualization Scheimpflug technology. The medical records of 50 eyes from 50 patients who received phacoemulsification and intraocular lens implantation because of age-related factors between June 2014 and September 2014 were retrospectively analyzed. FLACS was used in 12 eyes (FLACS group), and conventional phacoemulsification in 38 eyes (PHACO group). The evaluation of corneal biomechanical parameters included the first/second applanation time (A-time1/A-time2), the first/second applanation length (A-length1/A-length2), corneal velocity during the first/second applanation moment (Vin/Vout), highest concavity time, highest concavity-radius (HC-radius), peak distance (PD), deformation amplitude (DA), central corneal thickness (CCT), and intraocular pressure (IOP). The differences in A-length1/A-length2, IOP, CCT, $\mathrm{PD}$, and DA were significant in the PHACO group between those before, 1 week after, and 1 month after surgery. No significant differences in corneal biomechanical parameters were found between those at 1 month after surgery and before surgery. There were significant differences in IOP and CCT in the FLACS group between those before, 1 week after, and 1 month after surgery. There were no significant differences in the other corneal biomechanical parameters. No significant differences were found in corneal biomechanical parameters between those 1 month after surgery and before surgery. There were significant differences in A-length1/A-length2, CCT, PD, and DA between the two groups at 1 week after surgery. There
\end{abstract}

Correspondence to: Dr Hui Song, Tianjin Eye Hospital, Tianjin Key Laboratory of Ophthalmology and Visual Science, Tianjin Eye Institute, Clinical College of Ophthalmology, Tianjin Medical University, 4 Gansu Road, Heping, Tianjin 300020, P.R. China

E-mail: stn18m@163.com

\section{${ }^{*}$ Contributed equally}

Key words: corneal biomechanics, phacoemulsification, femtosecond laser were no significant differences in corneal biomechanical parameters between the two groups at 1 month after surgery. In conclusion, the effect of FLACS on corneal biomechanics is smaller than that of phacoemulsification. The corneal biomechanical parameters are restored to preoperative levels with the healing of the incision, and the reduction of swelling of the tissue near the incision.

\section{Introduction}

Femtosecond laser-assisted cataract surgery (FLACS), which includes lens lens fragmentation, clear corneal incisions, and limbal relaxing incisions, was first reported by Nagy et al (1) in 2009. The clinical application of the femtosecond laser has led to new developments for cataract surgery. The femtosecond laser system can make ladder-like multi-plane incisions in which the inner surface is enclosed. That is, the ladder between the corneal surface and matrix is made first to improve the impermeability of the incision, maintain intraocular pressure (IOP) and anterior chamber stability, and prevent leakage of aqueous humor, thus reducing the incidence of endophthalmititis. The femtosecond laser system was first used to complete the phacoemulsification steps including capsulorhexis, nucleus fragmentation, and clear corneal incision. The patients were then subjected to phacoemulsification to complete the entire cut along the tunnel made in former steps using a puncture knife (1). Masket et al (2) showed that the clear corneal incision made by FLACS had good impermeability and predictability.

In the present study, the effects of FLACS and phacoemulsification on corneal biomechanics were evaluated by corneal visualization Scheimpflug technology (Corvis ST).

\section{Patients and methods}

Patients. The medical records of 50 eyes from 50 patients who received phacoemulsification and intraocular lens (IOL) implantation because of age-related factors at the Tianjin Eye Hospital (Tianjin, China) from June 2014 to September 2014 were retrospectively analyzed. Inclusion criteria for the study were: axial length measured by IOL-Master was $22-24 \mathrm{~mm}$ before surgery, and corneal astigmatism $\leq 1.5 \mathrm{D}$. Exclusion criteria for the study were: pupil $<5 \mathrm{~mm}$ after mydriasis; patients with uveitis, glaucoma, retinal detachment, and 
Table I. Comparison of baseline parameters between the two groups.

\begin{tabular}{lccccc}
\hline & \multicolumn{4}{c}{ No. of cases } & \\
\cline { 2 - 4 } Groups & Eye & Eye $^{\mathrm{a}}$ (right/left) & $\mathrm{Sex}^{\mathrm{a}}$ (male/female) & Age $^{\mathrm{b}}$ (mean $\pm \mathrm{SD}$, years) & Axial length ${ }^{\mathrm{b}}(\mathrm{mean} \pm \mathrm{SD}, \mathrm{mm})$ \\
\hline FLACS group & 12 & $7 / 5$ & $4 / 8$ & $63.79 \pm 7.38$ & $23.12 \pm 2.45$ \\
PHACO group & 38 & $18 / 20$ & $15 / 23$ & $67.21 \pm 9.34$ & $22.98 \pm 2.69$ \\
$\chi^{2} / \mathrm{t}$ test & & 0.439 & 0.181 & 1.289 & 0.967 \\
P-value & & 0.530 & 1.261 & 2.342 & 3.819 \\
\hline
\end{tabular}

${ }^{\mathrm{a}} \chi^{2}$ test, ${ }^{\mathrm{b}} \mathrm{t}$-test. FLACS, femtosecond laser-assisted cataract surgery.

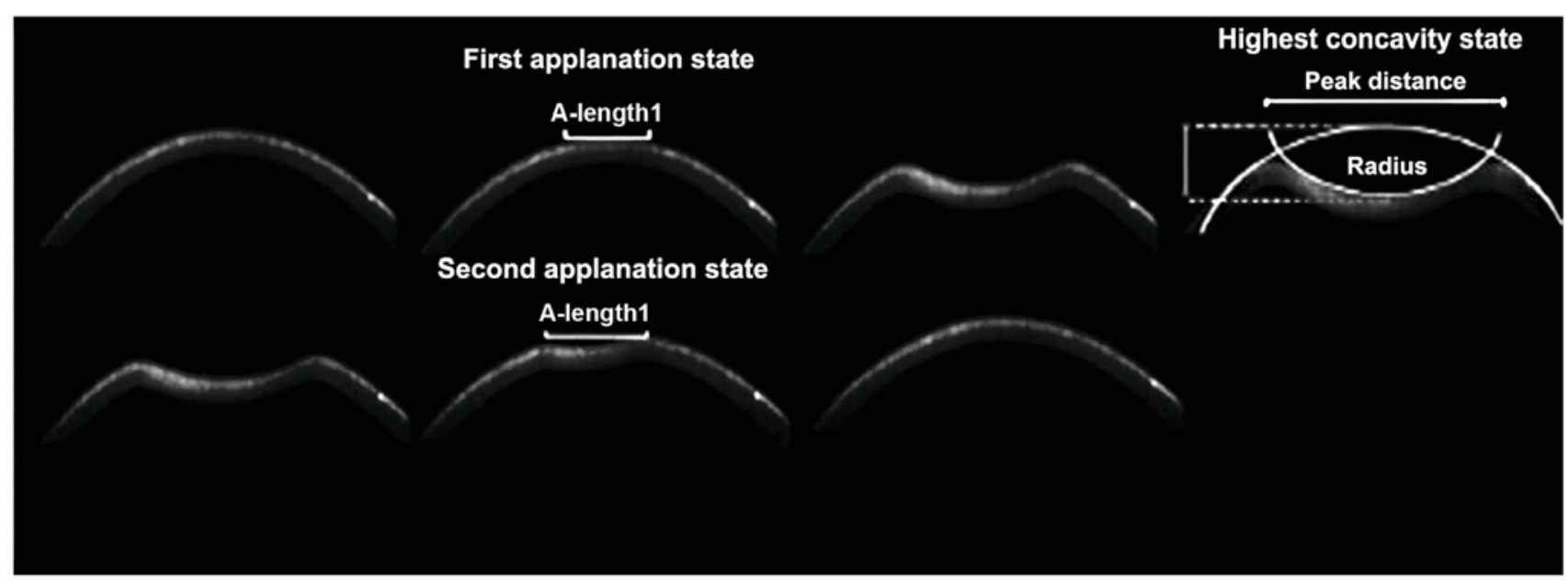

Figure 1. The entire process of corneal deformation by airflow used in Corvis ST. Corvis ST, corneal visualization Scheimpflug technology; A-length1/Alength2, first/second applanation length.

corneal leukoplakia; and patients with diabetes, hypertension, and coagulopathy. The patients were divided into the FLACS and PHACO groups according to the different surgical methods applied. Postoperative follow-up records included slit-lamp microscopy, and corneal biomechanics before and after surgery. The baseline parameters of the two groups are shown in Table I.

Surgical methods. Surgical procedures were performed by the same physician. Tropicamide eye drops (Santen Pharmaceutical Co., Ltd., Osaka, Japan) were used for mydriasis $30 \mathrm{~min}$ before surgery, and cimetidine hydrochloride eye drops (Alcon, Fort Worth, TX, USA) were used for surface anesthesia. A 3.0-mm clear corneal tunnel incision was made above the tempel or the nasal of the left eyes with a scalpel for patients in the PHACO group. Continuous circular capsulorhexis were made, and water was fully separated. The lens was removed by phacoemulsification. After polishing, IOLs were placed to the right position, and the incision was sealed. The FLACS group was treated with a LenSx femtosecond laser system (Alcon) for circular capsulorhexis, lens fragmentation, and clear corneal incision. A 3.0-mm clear corneal tunnel incision was made above the bitamporal or left part of the nose. The lens was removed by phacoemulsification. After polishing, IOLs were placed to the right position, and the incision was sealed. All the surgical procedures were successful and no complications occurred.
Biomechanical measurement of corneas. Corneal biomechanical parameters including the first/second applanation time (A-time1/A-time2), the first/second applanation length (A-length1/A-length2), corneal velocity during the first/second applanation moment (Vin/Vout), highest concavity time, highest concavity-radius (HC-radius), peak distance (PD), deformation amplitude (DA), central corneal thickness (CCT), and IOP were evaluated by Corvis ST. Corvis ST quantifies corneal biomechanical changes caused by corneal incision using the above biomechanical indicators to achieve a more objective analysis (3). Air pulse compression technology was used in Corvis ST to induce central corneal compression and deformation. Simultaneously, high-speed (4,330 frames/sec) shooting was performed according to the ultra-high Scheimpflug principle to dynamically record the entire process of deformation of the corneal central horizontal section (Fig. 1). Slow motion is presented on the display after analysis with software. The average value of three repeats was calculated to reduce errors.

Statistical analysis. SPSS 17.0 statistical software (Chicago, IL, USA) was used for data analysis. Qualitative data are expressed in terms of frequency, and quantitative data of measurement indexes are expressed as mean $\pm \mathrm{SD}$. The differences in the number of eyes, and sex between the PHACO and FLACS groups were analyzed by $\chi^{2}$ test. Paired $\mathrm{t}$-test was used for the comparison of corneal biomechanical 
Table II. Comparison of the corneal biomechanical parameters in the PHACO group before surgery, 1 week after surgery, and 1 month after surgery (mean $\pm \mathrm{SD})$.

\begin{tabular}{|c|c|c|c|c|c|c|}
\hline Variables & Before surgery & $\begin{array}{c}\text { Afer } 1 \text { week } \\
\text { of surgery }\end{array}$ & $\begin{array}{l}\text { After } 1 \text { month } \\
\text { of surgery }\end{array}$ & p1 & p2 & p3 \\
\hline A-length1 (mm) & $1.75 \pm 0.05$ & $1.87 \pm 0.05$ & $1.76 \pm 0.03$ & 0.039 & 0.645 & 0.038 \\
\hline A-length2 (mm) & $1.88 \pm 0.15$ & $1.55 \pm 0.32$ & $1.80 \pm 0.31$ & 0.017 & 0.717 & 0.024 \\
\hline $\operatorname{Vin}(\mathrm{m} / \mathrm{sec})$ & $0.15 \pm 0.03$ & $0.14 \pm 0.02$ & $0.15 \pm 0.02$ & 0.429 & 1.000 & 0.429 \\
\hline Vout (m/sec) & $-0.37 \pm 0.04$ & $-0.38 \pm 0.08$ & $-0.33 \pm 0.09$ & 0.800 & 0.134 & 0.083 \\
\hline IOP (mmHg) & $15.54 \pm 2.47$ & $17.91 \pm 3.30$ & $14.83 \pm 2.85$ & 0.047 & 0.157 & 0.035 \\
\hline Corrected IOP (mmHg) & $15.06 \pm 2.36$ & $17.72 \pm 3.33$ & $14.35 \pm 2.68$ & 0.042 & 0.147 & 0.039 \\
\hline $\mathrm{PD}(\mathrm{mm})$ & $4.11 \pm 1.19$ & $5.82 \pm 0.81$ & $4.51 \pm 1.12$ & 0.018 & 0.350 & 0.023 \\
\hline HC-radius (mm) & $7.12 \pm 0.56$ & $7.03 \pm 0.58$ & $7.24 \pm 0.74$ & 0.628 & 0.068 & 0.324 \\
\hline $\mathrm{DA}(\mathrm{mm})$ & $1.13 \pm 0.19$ & $1.62 \pm 0.16$ & $1.10 \pm 0.13$ & 0.006 & 0.631 & 0.012 \\
\hline $\mathrm{CCT}(\mu \mathrm{m})$ & $562.08 \pm 23.08$ & $589.83 \pm 26.26$ & $561.42 \pm 28.00$ & 0.016 & 0.950 & 0.009 \\
\hline A-time1 (msec) & $7.48 \pm 0.23$ & $7.40 \pm 0.30$ & $7.36 \pm 0.27$ & 0.458 & 0.271 & 0.715 \\
\hline A-time2 (msec) & $21.36 \pm 0.80$ & $21.47 \pm 0.40$ & $21.74 \pm 0.44$ & 0.661 & 0.119 & 0.255 \\
\hline Maximum crush time (msec) & $0.50 \pm 0.14$ & $0.58 \pm 0.17$ & $0.83 \pm 0.25$ & 0.309 & 0.754 & 0.194 \\
\hline
\end{tabular}

p1, comparison between before and 1 week after surgery; 2 , comparison between before and 1 month after surgery; p3, comparison between 1 week after surgery and 1 month after surgery. A-length1/A-length2, first/second applanation length; Vin/Vout, first/second applanation moment; IOP, intraocular pressure; PD, peak distance; HC-radius, highest concavity-radius; DA, deformation amplitude; CCT, central corneal thickness; A-time1/A-time2, first/second applanation time.

parameters between different time points within the same group. Independent-samples t-test was used for the comparison of corneal biomechanical parameters at the same time points between different groups.

\section{Results}

Comparison of the corneal biomechanical parameters in the PHACO group before surgery, 1 week after surgery, and 1 month after surgery. The differences in A-length1/A-length2, IOP, Corrected IOP, CCT, PD, and DA were statistically significant in the $\mathrm{PHACO}$ group between 1 week after and before surgery $(\mathrm{P}=0.039, \mathrm{P}=0.017, \mathrm{P}=0.047, \mathrm{P}=0.042, \mathrm{P}=0.018$, $\mathrm{P}=0.006, \mathrm{P}=0.016)$. There were no significant differences in the other corneal biomechanical parameters $(P>0.05)$. No significant differences were found in corneal biomechanical parameters between 1 month after and before surgery $(\mathrm{P}>0.05)$. The differences in A-length1/A-length2, IOP, Corrected IOP, CCT, PD, DA, and CCT were statistically significant in the PHACO group between 1 week after surgery and 1 month after surgery $(\mathrm{P}=0.038, \mathrm{P}=0.024, \mathrm{P}=0.035, \mathrm{P}=0.039, \mathrm{P}=0.023$, $\mathrm{P}=0.012, \mathrm{P}=0.09)$. There were no significant differences in the other corneal biomechanical parameters $(\mathrm{P}>0.05)$ (Table II).

Comparison of the corneal biomechanical parameters in the FLACS group before surgery, 1 week after surgery, and 1 month after surgery. There were significant differences in IOP, Corrected IOP and CCT in the FLACS group between 1 week after surgery and before surgery $(\mathrm{P}=0.043, \mathrm{P}=0.041$, $\mathrm{P}=0.035)$. No significant differences in the other corneal biomechanical parameters were found. No significant differences were found in corneal biomechanical parameters between 1 month after surgery and before surgery $(\mathrm{P}>0.05)$. There were significant differences in IOP, Corrected IOP and CCT in the FLACS group between 1 week after surgery and 1 month after surgery $(\mathrm{P}=0.042, \mathrm{P}=0.037, \mathrm{P}=0.044)$. No significant differences were found in the other corneal biomechanical parameters $(\mathrm{P}>0.05)$ (Table III).

Comparison of the corneal biomechanical parameters between the PHACO and FLACS groups before surgery, 1 week after surgery, and 1 month after surgery. There were significant differences in A-length1/A-length2, CCT, PD, and DA between the two groups at 1 week after surgery $(\mathrm{P}=0.041, \mathrm{P}=0.025$, $\mathrm{P}=0.016, \mathrm{P}=0.025, \mathrm{P}=0.047)$. There were no significant differences in the other corneal biomechanical parameters between the two groups $(\mathrm{P}>0.05)$. There were no significant differences in corneal biomechanical parameters between the two groups at 1 month after surgery (P>0.05) (Fig. 2).

\section{Discussion}

The cornea has non-linear, viscoelastic, anisotropic, and other biomechanical properties (4-7). The cornea, which is the most important structure of the refractive system, accounts for $70 \%$ of the total refractive power in humans. Therefore, the integrity of its organizational structure and the stability of its biomechanics are both essential for imaging. The cornea can be surgically modified to obtain the desired refractive power. The cornea is located at the 1/6 forefront of the eyeball. Given the special physiological location and physical and chemical properties of the cornea, it serves as the entry site of many surgical approaches. Surgical procedures, especially surgical incisions on corneal injuries, inevitably damage the integrity 
Table III. The comparison of corneal biomechanical parameters in the FLACS group before surgery, 1 week after surgery, and 1 month after surgery (mean $\pm \mathrm{SD})$.

\begin{tabular}{|c|c|c|c|c|c|c|}
\hline & Before surgery & $\begin{array}{l}\text { After } 1 \text { week } \\
\text { of surgery }\end{array}$ & $\begin{array}{l}\text { After } 1 \text { month } \\
\text { of surgery }\end{array}$ & $\mathrm{p} 1$ & $\mathrm{p} 2$ & p3 \\
\hline A-length1 (mm) & $1.77 \pm 0.05$ & $1.78 \pm 0.05$ & $1.76 \pm 0.04$ & 0.839 & 0.845 & 0.838 \\
\hline A-length2 (mm) & $1.86 \pm 0.17$ & $1.85 \pm 0.32$ & $1.82 \pm 0.30$ & 0.817 & 0.717 & 0.724 \\
\hline $\operatorname{Vin}(\mathrm{m} / \mathrm{sec})$ & $0.14 \pm 0.03$ & $0.14 \pm 0.02$ & $0.15 \pm 0.02$ & 0.829 & 1.000 & 0.829 \\
\hline Vout $(\mathrm{m} / \mathrm{sec})$ & $-0.36 \pm 0.04$ & $-0.37 \pm 0.08$ & $-0.34 \pm 0.09$ & 0.800 & 0.334 & 0.583 \\
\hline IOP (mmHg) & $14.72 \pm 2.25$ & $16.98 \pm 2.30$ & $14.53 \pm 2.74$ & 0.043 & 0.357 & 0.042 \\
\hline Corrected IOP (mmHg) & $15.14 \pm 2.33$ & $17.64 \pm 2.73$ & $14.66 \pm 2.56$ & 0.041 & 0.225 & 0.037 \\
\hline $\mathrm{PD}(\mathrm{mm})$ & $4.22 \pm 1.08$ & $4.33 \pm 1.09$ & $4.45 \pm 1.12$ & 0.468 & 0.350 & 0.323 \\
\hline HC-radius (mm) & $7.15 \pm 0.65$ & $7.10 \pm 0.68$ & $7.24 \pm 0.72$ & 0.715 & 0.412 & 0.308 \\
\hline $\mathrm{DA}(\mathrm{mm})$ & $1.14 \pm 0.17$ & $1.16 \pm 0.16$ & $1.13 \pm 0.14$ & 0.567 & 0.722 & 0.802 \\
\hline $\mathrm{CCT}(\mu \mathrm{m})$ & $556.22 \pm 22.06$ & $578.41 \pm 24.15$ & $560.42 \pm 23.79$ & 0.035 & 0.850 & 0.044 \\
\hline A-time1 (msec) & $7.42 \pm 0.25$ & $7.46 \pm 0.31$ & $7.36 \pm 0.29$ & 0.745 & 0.837 & 0.778 \\
\hline A-time2 (msec) & $21.62 \pm 0.81$ & $21.49 \pm 0.53$ & $21.75 \pm 0.63$ & 0.652 & 0.889 & 0.755 \\
\hline Maximum crush time (msec) & $0.56 \pm 0.12$ & $0.62 \pm 0.18$ & $0.71 \pm 0.21$ & 0.410 & 0.583 & 0.249 \\
\hline
\end{tabular}

p1, comparison between before and 1 week after surgery; 2 , comparison between before and 1 month after surgery; p3, comparison between 1 week after surgery and 1 month after surgery. FLACS, femtosecond laser-assisted cataract surgery; A-length1/A-length2, first/second applanation length; Vin/Vout, first/second applanation moment; IOP, intraocular pressure; PD, peak distance; HC-radius, highest concavity-radius; DA, deformation amplitude; CCT, central corneal thickness; A-time1/A-time2, first/second applanation time.

of the cornea. The corneal biomechanical properties also change, which in turn affect the postoperative refractive status.

Cataract surgery has gradually changed from vision rehabilitation to refractive surgery, which aims to achieve the best visual quality with minimal surgical trauma. With improvements of surgical techniques, postoperative corneal astigmatism has become a key factor affecting postoperative visual quality (8). Surgical astigmatism is caused by many factors, of which surgical incision is the main factor. The application of phacoemulsification on a clear corneal incision can cause slight reverse astigmatism, suggesting that the healing process of clear corneal incision directly affects changes of corneal astigmatism. This astigmatism is caused by the structure of the corneal incision, and corneal biomechanical changes (8). Thus, changes in corneal biomechanics can cause changes in corneal refractive status.

The measurement of corneal biomechanics can be used to quantitatively analyze corneal intrinsic properties, and to establish a more stereoscopic corneal model, to guide the preparation of corneal contact lenses, optimize the steps of corneal refractive surgery, and provide a theoretical basis for early diagnosis and treatment of keratoconus, glaucoma, large cornea, small cornea, and corneal flat and other diseases (8). The measurement of corneal biomechanical properties includes in vitro and in vivo measurements. The in vivo measurement method is based on the Reichert Ocular Response Analyzer (ORA). The major corneal biomechanical parameters measured by ORA include corneal hysteresis $(\mathrm{CH})$ and corneal resistance factor (CRF). $\mathrm{CH}$ is an indicator of energy absorption and dispersion capacity of the cornea. The $\mathrm{CH}$ value is affected by corneal thickness, hardness, hydration, and other factors. Greater $\mathrm{CH}$ value indicates greater ability of the cornea to resist external forces, which is important for clinical evaluation of the nature of the cornea (8). CRF, which is the overall hardness of the cornea, reflects the cumulative effect of corneal deformation caused by compression, including viscous resistance and elastic resistance (8). Song et al used ORA to assess the changes in corneal biomechanics before and after 4 weeks of phacoemulsification, where $\Delta \mathrm{CH}=-0.45 \pm 1.27 \mathrm{mmHg}$, $\Delta \mathrm{CRF}=-0.88 \pm 1.1 \mathrm{mmHg}, \Delta \mathrm{IOPg}=-1.58 \pm 3.15 \mathrm{mmHg}$, and $\Delta \mathrm{IOPcc}=-1.45 \pm 3.93 \mathrm{mmHg}(9)$. Greater CCT is accompanied with greater CRF value, and longer axial length is associated with a smaller drop in IOPcc, indicating that phacoemulsification can cause changes in corneal biomechanical properties including corneal epithelial features, corneal geometry, and corneal measurement $(10,11)$. In addition, Hager et al studied phacoemulsification on clear corneal incisions using ORA (12). It was found that CCT was increased from $556.8 \pm 32.5$ to $580.3 \pm 45.5 \mu \mathrm{m}$ after surgery, $\mathrm{CH}$ was reduced from $10.4 \pm 2.5$ to $9.2 \pm 1.9 \mathrm{mmHg}$ after surgery, and non-invasive IOP was increased from $17.9 \pm 3.8$ to $20.1 \pm 6.3 \mathrm{mmHg}$ after surgery. There was no significant difference in CCT and $\mathrm{CH}$ compared with the control group at 27 weeks after surgery. $\mathrm{CH}$ value was negatively correlated with IOP. Combined with other factors such as increased CCT caused by corneal edema after phacoemulsification, early postoperative anterior chamber inflammatory factors, blocked aqueous efflux, and other factors, transient IOP increase can occur after surgery. Similar results were found by Kamiya et al (13). Alió et al used ORA to study changes of corneal biomechanics after coaxial phacoemulsification and micro-incision phacoemulsification (14). The results showed that the corneal biomechanics were more stable after micro-incision phacoemulsification compared with those after standard coaxial phacoemulsification. 

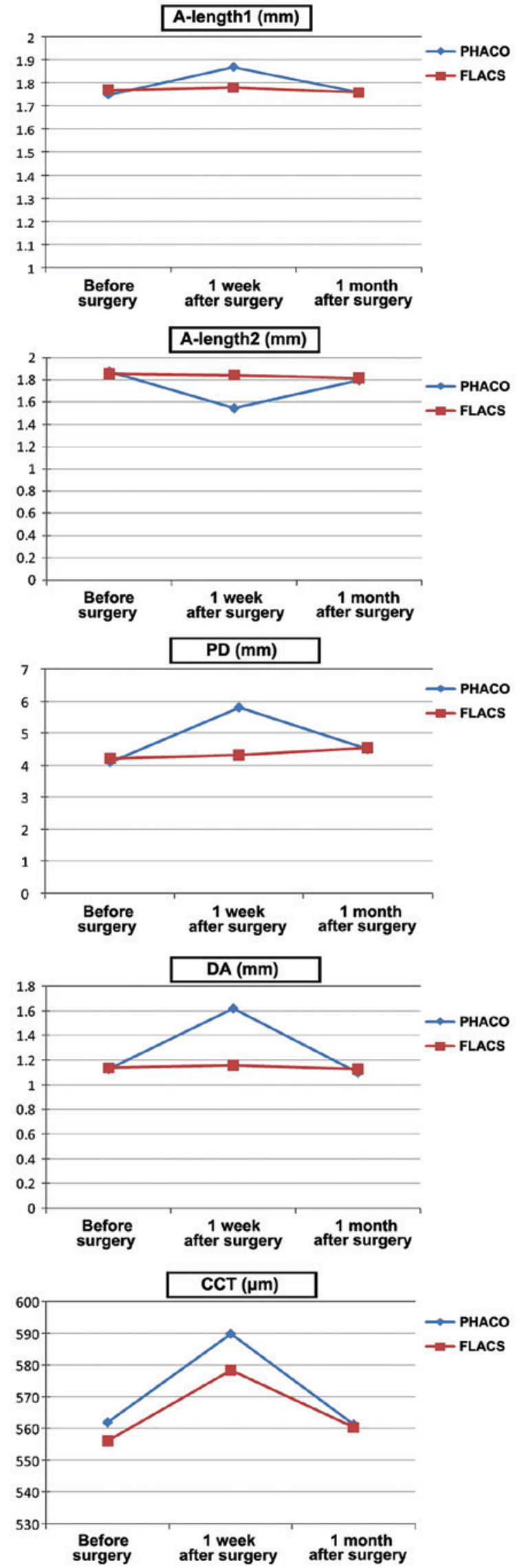

Figure 2. There were significant differences in A-length1/A-length2, CCT, $\mathrm{PD}$, and $\mathrm{DA}$ between the two groups at 1 week after surgery $(\mathrm{P}=0.041$, $\mathrm{P}=0.025, \mathrm{P}=0.016, \mathrm{P}=0.025, \mathrm{P}=0.047$ ). A-length1/A-length2, first $/$ second applanation length; CCT, central corneal thickness; PD, peak distance; DA deformation amplitude.
However, previous findings have shown that the measurement parameters of ORA ( $\mathrm{CH}$ and $\mathrm{CRF}$ ) have not yet formed a one-to-one correspondence with classical corneal biomechanical parameters. Therefore, corneal biomechanical properties cannot be expressed intuitively and fully $(15,16)$. In addition, the deformation process of the cornea cannot be displayed in real time $(14,17)$. Corvis ST is a new type of instrument for assessing the biomechanical properties of the cornea that records the corneal compression process in real time by Scheimpflug high-speed imaging technology. The computer system measures and records corneal biomechanical parameters, including: A-time1/A-time2, the time from the initial state to the flattened state/the time from maximum deformation to the time point when the cornea returns to the central level. This parameter is related to the calculation of IOP; A-length $1 /$ A-length 2 is the length of the flattened portion of the anterior cornea of the first/second flat state. This measurement index is affected by local fluctuation of the cornea; Vin/Vout is the instantaneous speed of corneal vertex downward/upward movement of the first/second flattened state. This parameter is a vector index, with a positive value representing a downward depression direction. A negative value indicates an upward bounce direction; highest concavity time is the time elapsed from the initial state to the maximum depression, and as shown in a previous study, the highest concavity time was the only age-related biomechanical parameter (18). Additionally, $\mathrm{HC}$-radius is the radius of curvature of the corneal anterior surface when the cornea is at the largest deformation status; PD is the horizontal distance between the highest points of the corneal non-deformed part at the maximum depression status; DA is the vertical shift of the corneal apex from the initial state to the maximum depression shift, which is one of the most important corneal biomechanical parameters (3). Additionally, CCT is the vertical distance of the anterior and posterior cornea at the apex of the cornea, measured based on the central horizontal section at the initial state. Corvis ST also obtains IOP based on the pulse pressure and A-time1/Atime2. In addition, Corvis ST provides corrected IOP based on corneal thickness. The corneal biomechanical parameters recorded by Corvis ST are the parameters at the deformed nodes, which can be used to specifically analyze changes in corneal biomechanics (3). These parameters obtained by Corvis ST, both in image analysis and in biomechanics, have not been adequately described in the literature, although these parameters have demonstrated good reproducibility and consistency $(19,20)$.

At present, Corvis ST has notbeen reported to assess changes in corneal biomechanics before and after phacoemulsification. The femtosecond laser technology in corneal refractive surgery has been widely applied in clinic, and corneal biomechanical evaluation of femtosecond laser refractive surgery has been reported (21). To compare the effect of femtosecond laser flap and mechanical knife flap on corneal biomechanics in LASIK, the corneal flap produced by the femtosecond laser was more flat and uniform in thickness (22). In addition, the diameter of the mechanical knife flap is affected by corneal curvature and other corneal morphological factors, leading to significant individual differences (23). The femtosecond laser made the corneal flap more regular, and the femtosecond laser can control the biomechanical changes caused by the flap to 
a better degree (24). Femtosecond laser surgery can induce more obvious early inflammation (25), and more fibrotic scars can be observed in the peripheral area by confocal microscopy (26), which are conducive to the recovery of corneal biomechanics. Corneal biomechanics change significantly after corneal refractive surgery, and femtosecond lasers can be used in corneal refractive surgery to achieve more stable corneal biomechanics (27). In the present study, Corvis ST was used to compare changes of corneal biomechanics between the application of PHACO and FLACS on corneal incision before and after surgery, and to further analyze the effects of the two methods on corneal biomechanics.

The differences in A-length1/A-length2, IOP, Corrected IOP, CCT, PD, and DA were statistically significant in the PHACO group between those at 1 week after surgery and before surgery $(\mathrm{P}=0.039, \mathrm{P}=0.017, \mathrm{P}=0.047, \mathrm{P}=0.042, \mathrm{P}=0.018, \mathrm{P}=0.006$, $\mathrm{P}=0.016)$. There were no significant differences in the other corneal biomechanical parameters $(\mathrm{P}>0.05)$. A-length1 value was increased at 1 week after surgery compared with that before surgery. A possible explanation is that corneal integrity was destroyed, and the elasticity was reduced. When the external pressure exerts a force on the anterior surface of the cornea, the ability of the cornea to resist this force becomes lower, resulting in a larger value. A-length 2 is the length of the flattening part of the corneal anterior surface at the moment the cornea is turning from the concave surface to the convex surface. All the forces involved in this process include the inherent viscoelasticity of the cornea, IOP, and the gradually reduced air flow. The increased value of A-length 2 at 1 week after surgery may have been caused by the destroyed impermeability of the cornea. The CCT value was increased at 1 week after surgery possibly because of the application of ultrasound energy and corneal edema. In addition, the positive correlation between IOP and CCT can explain the increased IOP at 1 week after surgery. PD and DA values were increased at 1 week after surgery compared with those before surgery, possibly because of the reduced integrity and impermeability. There was no significant difference in HC-radius value before and 1 week after surgery, which can be explained by the increase of both PD and DA values. No significant differences were found in A-time1/A-time2, $\mathrm{Vin} /$ Vout, or highest concavity time before and 1 week after surgery $(\mathrm{P}>0.05)$. A possible explanation is that the inside and outside forces can be balanced by the viscoelasticity of the cornea. Therefore, the value at the node position did not change significantly. No significant differences were found in corneal biomechanical parameters between those before and 1 month after surgery $(\mathrm{P}>0.05)$ possibly because of the healing of clear corneal incisions and the relieved corneal edema.

The femtosecond laser is a near infrared light with a wavelength of $1,053 \mathrm{~nm}$. The femtosecond laser interacts with tissue in the form of a pulse to produce plasma. The plasma fusion expands and separates the tissue by photolysis, resulting in precise tissue cutting at the molecular degree, which greatly reduces damage to non-targeted tissues. There were significant differences in IOP, Corrected IOP and CCT in the FLACS group between those before and 1 week after surgery $(\mathrm{P}=0.043$, $\mathrm{P}=0.041, \mathrm{P}=0.035$ ), although no significant differences in the other corneal biomechanical parameters were found $(\mathrm{P}>0.05)$. The main side incision made by the femtosecond laser, nucleus fragmentation, and the use of ultrasonic energy, caused corneal edema. This in turn increased CCT value, and IOP and CCT were positively correlated. With the reduced corneal edema and corneal wound healing, CCT and IOP were restored to preoperative levels at 1 month after surgery.

There were significant differences in A-length1/A-length2, CCT, PD, and DA between the two groups at 1 week after surgery $(\mathrm{P}=0.041, \mathrm{P}=0.025, \mathrm{P}=0.016, \mathrm{P}=0.025, \mathrm{P}=0.047)$. This indicated that corneal incisions made in the FLACS group could maintain corneal integrity to a better degree than corneal incisions made in the PHACO group, leading to smaller corneal biomechanical changes. The smaller CCT values may be related to less ultrasound energy and less effective time applied in the FLACS group. With the healing of corneal incisions, and the regression of corneal edema, no significant differences were found in corneal biomechanical parameters between the two groups at 1 month after surgery $(\mathrm{P}>0.05)$.

In conclusion, compared with corneal incisions made with a puncture knife, the incisions made by femtosecond laser can lead to better stability at the early stage after surgery, and have less impact on corneal biomechanics. The posterior corneal wrinkles, the use of intraoperative viscoelastic agents, and corneal endothelium loss are important factors in postoperative corneal biomechanical changes (28). However, with reduced corneal edema and corneal incision healing, biomechanical parameters of the two groups returned to preoperative levels. In the present study, cataract patients with level II-III lens nucleus hardness were selected. The incidence of postoperative corneal edema and corneal endothelial cell loss were avoided as much as possible to reduce the corneal biomechanical effects. Future studies will focus on corneal biomechanical changes within the first day after surgery. The preparation of transparent corneal incisions will directly affect corneal biomechanical changes. The present study only compared the corneal biomechanical effects of 3.0-mm clear corneal incisions between the two groups. Examining the effects of different incision sites, incision shapes, and incision sizes on corneal biomechanics to provide theoretical guidance for the identification of the optimal corneal incision, to obtain optimal postoperative refractive status is imperative.

\section{Acknowledgements}

The present study was supported by a specialized research fund from Tianjin Application Foundation and Advanced Technology Program (15JCYBJC27600).

\section{References}

1. Nagy Z, Takacs A, Filkorn T and Sarayba M: Initial clinical evaluation of an intraocular femtosecond laser in cataract surgery. J Refract Surg 25: 1053-1060, 2009.

2. Masket S, Sarayba M, Ignacio T and Fram N: Femtosecond laser-assisted cataract incisions: architectural stability and reproducibility. J Cataract Refract Surg 36: 1048-1049, 2010.

3. Hon Y and Lam AK: Corneal deformation measurement using Scheimpflug noncontact tonometry. Optom Vis Sci 90: e1-e8, 2013.

4. Hayes S, Boote C, Lewis J, Sheppard J, Abahussin M, Quantock AJ, Purslow C, Votruba M and Meek KM: Comparative study of fibrillar collagen arrangement in the corneas of primates and other mammals. Anat Rec (Hoboken) 290: 1542-1550, 2007.

5. Boote C, Hayes S, Abahussin M and Meek KM: Mapping collagen organization in the human cornea: left and right eyes are structurally distinct. Invest Ophthalmol Vis Sci 47: 901-908, 2006. 
6. Meek KM and Boote C: The organization of collagen in the corneal stroma. Exp Eye Res 78: 503-512, 2004.

7. Aghamohammadzadeh H, Newton RH and Meek KM: X-ray scattering used to map the preferred collagen orientation in the human cornea and limbus. Structure 12: 249-256, 2004

8. Brown NA and Sparrow JM: Control of astigmatism in cataract surgery. Br J Ophthalmol 72: 487-493, 1988.

9. Song X, Langenbucher A, Gatzioufas Z, Seitz B and El-Husseiny M: Effect of biometric characteristics on the change of biomechanical properties of the human cornea due to cataract surgery. BioMed Res Int 2014: 628019, 2014

10. Spörl E, Terai N, Haustein M, Böhm AG, Raiskup-Wolf F and Pillunat LE: Biomechanical condition of the cornea as a new indicator for pathological and structural changes. Ophthalmologe 106: 512-520, 2009 (In German).

11. Wilson SE and Klyce SD: Quantitative descriptors of corneal topography. A clinical study. Arch Ophthalmol 109: 349-353, 1991.

12. Hager A, Loge K, Schroeder B, Füllhas MO and Wiegand W: Effect of central corneal thickness and corneal hysteresis on tonometry as measured by dynamic contour tonometry, ocular response analyzer, and Goldmann tonometry in glaucomatous eyes. J Glaucoma 17: 361-365, 2008.

13. Kamiya K, Shimizu K, Ohmoto F and Amano R: Time course of corneal biomechanical parameters after phacoemulsification with intraocular lens implantation. Cornea 29: 1256-1260, 2010.

14. Alió JL, Agdeppa MC, Rodríguez-Prats JL, Amparo F and Piñero DP: Factors influencing corneal biomechanical changes after microincision cataract surgery and standard coaxial phacoemulsification. J Cataract Refract Surg 36: 890-897, 2010.

15. McMonnies CW: Assessing corneal hysteresis using the Ocular Response Analyzer. Optom Vis Sci 89: E343-E349, 2012.

16. Detry-Morel M, Jamart J and Pourjavan S: Evaluation of corneal biomechanical properties with the Reichert Ocular Response Analyzer. Eur J Ophthalmol 21: 138-148, 2011.

17. Terai N, Raiskup F, Haustein M, Pillunat LE and Spoerl E: Identification of biomechanical properties of the cornea: the ocular response analyzer. Curr Eye Res 37: 553-562, 2012.

18. Valbon BF, Ambrósio R Jr, Fontes BM and Alves MR: Effects of age on corneal deformation by non-contact tonometry integrated with an ultra-high-speed (UHS) Scheimpflug camera. Arq Bras Oftalmol 76: 229-232, 2013.
19. Valbon BF, Ambrósio R Jr, Fontes BM, Luz A, Roberts CJ and Alves MR: Ocular biomechanical metrics by CorVis ST in healthy Brazilian patients. J Refract Surg 30: 468-473, 2014.

20. Nemeth G, Hassan Z, Csutak A, Szalai E, Berta A and Modis L Jr: Repeatability of ocular biomechanical data measurements with a Scheimpflug-based noncontact device on normal corneas. J Refract Surg 29: 558-563, 2013.

21. Hamilton DR, Johnson RD, Lee N and Bourla N: Differences in the corneal biomechanical effects of surface ablation compared with laser in situ keratomileusis using a microkeratome or femtosecond laser. J Cataract Refract Surg 34: 2049-2056, 2008.

22. Krueger RR and Dupps WJ Jr: Biomechanical effects of femtosecond and microkeratome-based flap creation: prospective contralateral examination of two patients. J Refract Surg 23: 800-807, 2007.

23. Gimbel HV, Iskander NG, Peters NT and Penno EA: Prevention and management of microkeratome-related laser in situ keratomileusis complications. J Refract Surg 16 (Suppl): 226-229, 2000.

24. Talamo JH, Meltzer J and Gardner J: Reproducibility of flap thickness with IntraLase FS and Moria LSK-1 and M2 microkeratomes. J Refract Surg 22: 556-561, 2006.

25. Kim JY, Kim MJ, Kim TI, Choi HJ, Pak JH and Tchah H: A femtosecond laser creates a stronger flap than a mechanical microkeratome. Invest Ophthalmol Vis Sci 47: 599-604, 2006.

26. Sonigo B, Iordanidou V, Chong-Sit D, Auclin F, Ancel JM, Labbé A and Baudouin C: In vivo corneal confocal microscopy comparison of intralase femtosecond laser and mechanical microkeratome for laser in situ keratomileusis. Invest Ophthalmol Vis Sci 47: 2803-2811, 2006.

27. Schmack I, Dawson DG, McCarey BE, Waring GO III, Grossniklaus HE and Edelhauser HF: Cohesive tensile strength of human LASIK wounds with histologic, ultrastructural, and clinical correlations. J Refract Surg 21: 433-445, 2005.

28. Kucumen RB, Yenerel NM, Gorgun E, Kulacoglu DN, Oncel B, Kohen MC and Alimgil ML: Corneal biomechanical properties and intraocular pressure changes after phacoemulsification and intraocular lens implantation. J Cataract Refract Surg 34: 2096-2098, 2008. 